Supporting Information

\title{
Graphene-layered Eggshell Membrane as a Flexible and Functional Scaffold for Enhanced Proliferation and Differentiation of Stem Cells
}

Sunho Park, ${ }^{\dagger+}$ Teayeop Kim,,${ }^{+}+$Yonghyun Gwon, ${ }^{\dagger}$ Sujin Kim,${ }^{\dagger}$ Daun Kim, ${ }^{\dagger}$ Hyun-Ha Park,${ }^{\S}$

Ki-Taek Lim, "Hoon Eui Jeong, ${ }^{\S, ~ *}$ Kyunghoon Kim,, , * and Jangho Kim", *

${ }^{\dagger}$ Department of Rural and Biosystems Engineering, Chonnam National University, Gwangju 61186, Republic of Korea

* School of Mechanical Engineering, Sungkyunkwan University, Suwon 16419, Republic of Korea

$\S$ Department of Mechanical Engineering, Ulsan National Institute of Science and Technology (UNIST), Ulsan 44919, Republic of Korea

"Department of Biosystems Engineering, College of Agricultural and Life Sciences, Kangwon National University, Chuncheon, 24341, Republic of Korea

* Corresponding authors: H.E. Jeong (hoonejeong@unist.ac.kr), K. Kim (kenkim@skku.edu), and J. Kim (rain2000@jnu.ac.kr) 

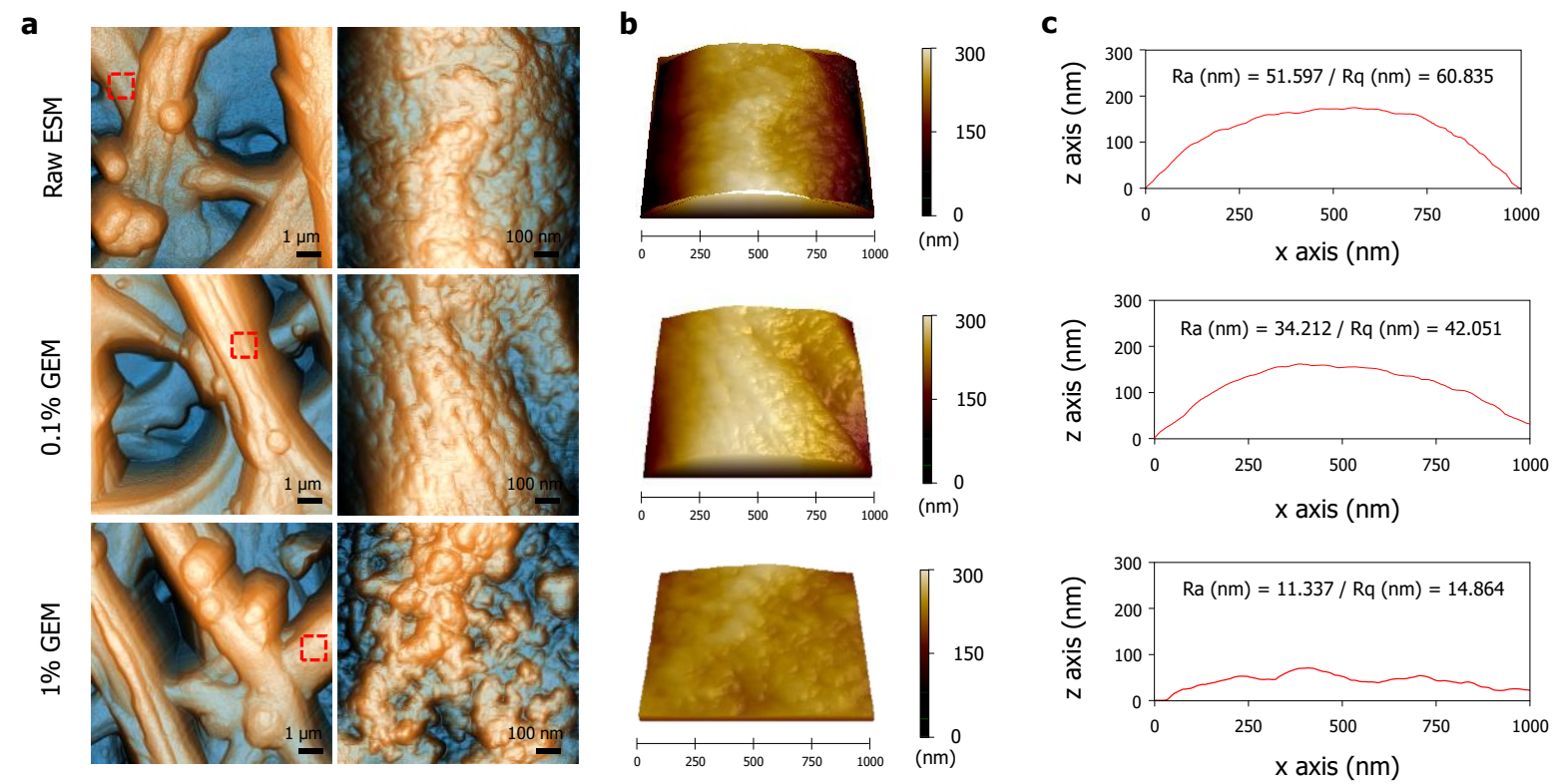

Figure S1. The atomic force microscope (AFM) images of the microfibers on the raw eggshell membrane (ESM) and graphene-layered ESM (GEM) scaffolds. (a) Representative AFM images with $10 \mu \mathrm{m} \times 10 \mu \mathrm{m}$ (left) and $1 \mu \mathrm{m} \times 1 \mu \mathrm{m}$ (right). (b) 3D-view of AFM images with $1 \mu \mathrm{m} \times 1 \mu \mathrm{m}$. (c) Line profile, Ra and Rq values of the raw ESM and GEM scaffolds. 